\title{
Parafoveal word processing during eye fixations in reading: Effects of word frequency
}

\author{
ALBRECHT WERNER INHOFF \\ University of New Hampshire, Durham, New Hampshire \\ and \\ KEITH RAYNER \\ University of Massachusetts, Amherst, Massachusetts
}

\begin{abstract}
The present experiment measured eye fixations in reading to determine whether word frequency affects the processing of the fixated word and the processing of the word to the right of the fixated word (the parafoveal word). In the experiment, subjects read sentences that contained either a critical high- or low-frequency target word. High- and low-frequency targets were matched on word length and a number of other variables. In one condition, parafoveal visual information to the right of the fixated word was denied or distorted; in other conditions, information about the parafoveal word to the right of the fixated word was available. The main results showed shorter fixations on high-frequency than on low-frequency target words. Furthermore, readers gained more effective previews from high-frequency parafoveal target words than from low-frequency parafoveal target words.
\end{abstract}

There are two major ways in which parafoveal information can be used during eye fixations in reading. First, it can be used to help readers determine where to look next, and second, it can be used to aid word-recognition processes. A number of studies have indicated that parafoveally obtained information assists eye guidance; specifically, when parafoveal word length information is denied during a fixation in reading, reading is hindered (McConkie \& Rayner, 1975; Pollatsek \& Rayner, 1982; Rayner \& Bertera, 1979; Rayner, Inhoff, Morrison, Slowiaczek, \& Bertera, 1981). Some controversy exists, however, on the role of parafoveal information in word recognition. On some fixations, the word to the right of the fixated word is identified and skipped on the subsequent saccade (Ehrlich \& Rayner, 1981; Rayner, Balota, \& Pollatsek, in press). When word skipping occurs, the duration of the fixation that precedes skipping can be related to the characteristics of the skipped word (Hogaboam, 1983). Yet, for content words, word skipping occurs on a minority of saccades (Hogaboam, 1983; Just \& Carpenter, 1980). How much useful information is obtained from a parafoveal word on the majority of fixations, when the parafoveal word is the target of the following fixation?

Rayner, Well, Pollatsek, and Bertera (1982) and Lima and Inhoff (1985) found that less viewing time was neces-

\footnotetext{
This research was supported by Grant IN 27/1-1 of the Deutsche Forschungsgemeinschaft to Albrecht Inhoff and by Grants HD 12727 and HD 17246 to Keith Rayner. We would like to thank Alexander Pollatsek, Charles Clifton, George McConkie, Susan Lima, and Ken Paap for their help on an earlier draft of the paper. Requests for reprints should be sent to Albrecht Inhoff, Department of Psychology, University of New Hampshire, Durham, NH 03824.
}

sary to process a word when the first three letters of a parafoveal word were available during each fixation than when such information was not available. Rayner et al. (1982) argued that the word's initial three-letter sequence is used during a fixation to speed the lexical processing of the parafoveal word during the following fixation of the word (see also Rayner, McConkie, \& Zola, 1980). However, on the basis of experiments in which the letters in a target-word location alternated with each eye movement (so that bears changed to peaks after one eye movement and then back to bears after the following eye movement), McConkie, Zola, Blanchard, and Wolverton (1982) concluded that information used to identify a word is obtained only on the fixation on which the word is completely identified.

More recent evidence (Balota, Pollatsek, \& Rayner, 1985) has demonstrated that partial-word information obtained from the parafovea is useful in identifying the word on the following fixation. Moreover, Balota et al. showed that the effective use of parafoveal information during the following fixation on the word is a function of the contextual constraints. Parafoveal information was used more effectively when the predictability of the parafoveal word was high than when the predictability was low.

Are there differences in the effective use of parafoveal information when prior contextual constraints are held constant? Erdmann and Dodge (cited in Huey, 1908/1968) found that highly familiar words could be identified farther from fixation than unfamiliar words. This suggests that factors intrinsic to parafoveal words can affect the use of parafoveal information during a fixation in reading. One well-studied word characteristic is word frequency, the effects of which are largely a result of word 
familiarity (Gernsbacher, 1984). Word frequency has been closely associated with lexical access in word recognition (Morton, 1969; Whaley, 1978; see Balota \& Chumbley, 1984, for a different view) so that high-frequency words are accessed faster than low-frequency words. Consistent with this position, high-frequency words are fixated for a shorter amount of time in reading than are lowfrequency words (Inhoff, 1984; Just \& Carpenter, 1980; Kliegl, Olson, \& Davidson, 1982; Rayner, 1977). Provided that word frequency affects the ease of lexical access, larger parafoveal preview benefits from highfrequency parafoveal words than from low-frequency parafoveal words would indicate that parafoveal previews are used to initiate lexical access. Kliegl et al. (1982) have pointed out, however, that studies that show shorter fixation times on high-frequency words than on lowfrequency words have confounded word frequency and word length, so that decreases in word frequency were accompanied by increases in word length. Some component of the viewing time that was associated with variations in word frequency could thus have resulted from variations in word length. Consequently, larger parafoveal preview benefits from high-frequency words than from low-frequency words could result from differences in word length if this factor is not controlled.

The goal of the present study was to test whether lexical properties of the parafoveal word could influence parafoveal processing. Specifically, we were interested in the extent to which the frequency of the parafoveal word would affect parafoveal preview benefits when effects of word length were eliminated.

In the experiment, subjects read sentences of the form

(1) His painful accident caused him trouble

or

(2) His painful fracture caused him trouble.

Targets (italicized) were high-frequency content words, for example, accident, in sentences of Type 1 and lowfrequency content words, for example, fracture, in sentences of Type 2 . Both high- and low-frequency words were identical in word length and were read under three different viewing conditions. In a one-word-window condition, all text to the left of fixation was displayed, but only letters of the currently fixated word were available to the right of fixation so that the range of effective parafoveal vision was defined by the right boundary of the fixated word. In a two-word-window condition, the currently fixated word (again including all text to the left) and the word immediately to its right were displayed during each fixation, thus extending the parafoveal preview one word into the parafovea. A full-line condition was also included in which no viewing limitations were imposed, so that the whole line of text was available during each fixation.

If readers obtain more effective parafoveal previews from high-frequency words than from low-frequency words, then word frequency and window size should inter- act. Relatively small benefits of word frequency should be obtained when parafoveal previews are denied, and relatively large benefits should accrue when previews are available. This interaction was sought in first-fixation durations and gaze durations on the target words. First fixations consisted of the initial fixation placed on a target word; gaze durations consisted of the cumulated viewing time on a critical word prior to a saccade away from the word. First fixations have been shown to be sensitive to the initial processing of a word (Inhoff, 1984; Lima \& Inhoff, 1985); gaze durations, which include refixations of the word, are more global indicators of word processing and are likely to include postlexical processing operations (Inhoff, 1984, 1985; Just \& Carpenter, 1980). We also analyzed saccade lengths to high- and low-frequency parafoveal targets to test whether word frequency affected the computation of the size of the eye movement to the target.

\section{METHOD}

\section{Subjects}

Twenty-four students at the University of Massachusetts were paid to participate in the experiment. All subjects had normal vision and were able to read without the aid of corrective lenses.

\section{Materials}

The materials consisted of 60 pairs of sentences of the form noun phrase-verb-noun phrase (NP-V-NP), in which the initial NP comprised the sequence article-adjective-noun. The two members of each pair of sentences were identical except for the noun of the initial NP, called the target, which was either a high-frequency or a low-frequency word. A sample of sentence pairs is shown in $\mathrm{Ta}$ ble 1 . High-frequency targets were words with frequency counts of more than 30 million (KuCera \& Francis, 1967); low-frequency targets were words with frequency counts of 25 or less per million. The average frequencies of high- and low-frequency targets were 130.6 and 8.1 per million, respectively. High- and lowfrequency words were identical in their number of constituent letters and contained between 3 and 8 letters. The two groups of words were matched on concreteness and number of syllables. Care was taken to make a given sentence beginning (article-adjective) neither more nor less contextually predictable of the high-frequency target than of the low-frequency target. To assess effects of prior context, that is, of the article and adjective preceding the target, we asked 16 subjects (none of whom participated in the reading experiment) to guess a likely continuation of the sentence's initial NP. An evaluation of these guesses showed that subjects correctly guessed the high- and low-frequency target on less than $1 \%$ of the trials, which indicates that the contextual constraints imposed on the target were relatively low.

The length of the words preceding and following the target ranged from 4 to 8 letters. This range was selected to increase the like-

Table 1

A Sample of the Sentences Used in the Experiment
The slow music/waltz captured her attention
The tired teacher/traitor left the room.
The captivating book/tale described his life.
A broken car/fir blocked the road.
The cold water/tonic tasted stale.
The handmade frame/quilt decorated the wall

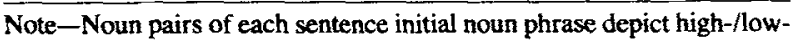
frequency target words. 
lihood that at least one fixation preceded and followed the fixation of the target (Rayner, 1979). Twelve practice sentences were added, the linguistic structure of which was identical to that of the experimental sentence pairs. Six of the practice sentences contained low-frequency nouns and six contained high-frequency nouns in their initial NPs. So that each sentence could be presented in a single line of text, no sentences were longer than 42 character spaces.

\section{Design}

Two lists of sentences were constructed, each containing $60 \mathrm{ex}$ perimental and 12 practice sentences. The experimental sentences of each list were identical, except that the high-frequency targets of the experimental sentences in one list were replaced with the corresponding low-frequency targets in the alternative list. Each list was segmented into three blocks of sentences, with each block consisting of 4 practice sentences followed by 20 experimental sentences. Half of the experimental sentences of each block contained high-frequency targets; the remaining half contained low-frequency targets. The succession of high- and low-frequency targets within a list was randomized. Each block of 24 sentences was presented in one of three presentation conditions, the sequence of which was counterbalanced across 3 successive subjects.

Three display conditions were used in the experiment. In the fullline condition, the entire line of text was available during each fixation. The two-word-window condition restricted the area from which actual text information could be obtained. In this condition, the word that was currently fixated, all text to the left of fixation, and the word immediately to the right of the fixated word were provided during each fixation. Parafoveal previews were further restricted in the one-word-window condition in which only the currently fixated word and text to the left of fixation were available during a fixation. For half of the subjects, all letters outside the experimentally determined window were replaced by confusable letters (e.g., the letters $b$ and $n$ were replaced by the letters $d$ and $\mathrm{m}$, respectively); for the remaining subjects, all letters outside the window were replaced by Xs. Interword blank spaces were maintained in both instances. Word list and type of parafoveal mutilation outside the window were varied between subjects; word frequency and window size were varied within subjects. First fixations and gaze durations, as well as saccade length to the target, were analyzed in 2 (word frequency) $\times 2$ (parafoveal mutilation) $\times 3$ (window size) analyses of variance.

\section{Apparatus}

In the experiment, the subject's eyes were $46 \mathrm{~cm}$ from a Hewlett Packard 1300 A cathode ray tube (CRT) that was used to present the sentences. The CRT had a $\mathrm{P}-31$ phosphor with the characteristic that removing a character resulted in a drop to $1 \%$ of maximum brightness within $.25 \mathrm{msec}$. Three character spaces of text equaled $1^{\circ}$ of visual angle. The sentences were presented in lower case, except for the first letter of the sentence. Luminance was adjusted to a comfortable viewing level; display intensity was occasionally reduced during the study as the reader became more dark adapted.

Eye-movement recording was accomplished with a Stanford Research Institute dual Purkinje eyetracker. The eyetracker had a resolution of $10^{\prime}$ of arc, and output was linear over the visual angle subtended by each sentence. The eyetracker and CRT were interfaced with a Hewlett Packard 2100A computer, which controlled the experiment. The signal from the eyetracker was sampled every millisecond by the computer. Each 4 msec of eyetracker output was compared with the output of the prior $4 \mathrm{msec}$ to determine whether the eye was fixated or in motion. The limiting factor of our equipment was the time needed to plot the display, which could be as much as $4 \mathrm{msec}$. Within $1 \mathrm{msec}$ after the plotting was completed, the eye position was read and a new display was computed and plotted conditional on eye position. The plotting thus occurred independently of whether the eye was judged to be in a saccade or in a fixation. The window was defined relative to the letter fixated, so that the window would remain the same when a letter was fixated even if there was a small movement of the eye that occurred within the space of a letter. Two successive eye fixations more than half a character apart marked the start of a saccade; two successive eye positions less than half a character apart marked the start of a new fixation. Testing of the display changes indicated that the changes associated with the completion of the saccade were completed within $5 \mathrm{msec}$.

\section{Procedure}

The subjects were tested individually. When a subject arrived, a bite bar was prepared that served to eliminate head movements during the experiment. Each subject received detailed instructions about the experimental procedure and was familiarized with the equipment. A calibration of the eye-tracking system began each session.

After calibration, three crosses were displayed at the left, center, and right of the screen. The subject's focal point was marked by a fourth cross that moved in synchrony with the reader's eyes. When the fourth cross superimposed itself over the three marked positions, as the subject sequentially fixated each position, the calibration was considered successful and the first practice sentence was displayed. Each sentence was read in the following manner: An initial fixation marker was displayed at the left side of the CRT; this position coincided with the first letter position of the first word on the line of text. The sentence was displayed by the experimenter as soon as the subject's central point of fixation was successfully placed on the left calibration marker. After reading a sentence, the subject pressed a button that replaced the sentence with the fixation marker. The subject was instructed to read each sentence for comprehension so that he or she would be able to paraphrase its content. Occasionally, readers were asked to repeat or to paraphrase a sentence immediately after reading it. This occurred on approximately $10 \%$ to $15 \%$ of the trials. All subjects were able to easily report the sentences.

\section{Scoring}

A target was considered fixated when a reader's point of fixation fell on one of its component letters or the blank space immediately preceding it. Trials in which the eyetracker lost track of the eye or in which a blink occurred were excluded from the analyses. Such artifacts occurred on less than $5 \%$ of the trials. The computer kept a complete record of the duration and location of each fixation, as well as the sequence of fixations.

\section{RESULTS AND DISCUSSION}

\section{Effects of Parafoveal Word Frequency on Fixation Durations and Saccade Length}

First fixation durations. First fixations were longer on low-frequency words (264 msec) than on highfrequency words $(248 \mathrm{msec})[F(1,20)=14.44$, $p<.002$ ], even though word length was held constant. Effects of window size were also significant; as expected, fixations were longer in the one-word-window condition $(275 \mathrm{msec})$ than in the two-word-window condition $(247 \mathrm{msec})$ or the full-line condition $(246 \mathrm{msec})[F(2,40)$ $=14.52, p<.001]$. The interaction of word frequency and window size was also significant $[F(2,40)=4.72$, $p<.025]$ and is shown in Figure 1. This interaction reveals that a parafoveal preview of a high-frequency word led to larger preview benefits than a parafoveal preview of a low-frequency word. Simple comparisons performed 


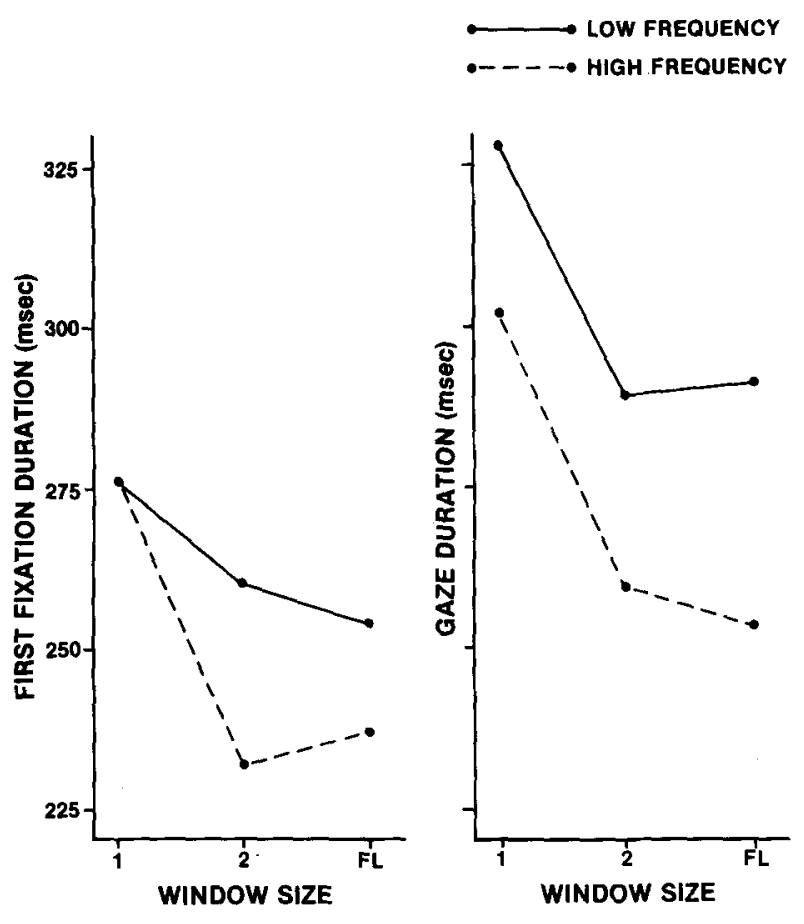

Figure 1. First-fixation durations (left panel) and gaze durations (right panel) as a function of window size and word frequency.

on the interaction showed significant parafoveal preview effects in all parafoveal preview conditions, regardless of whether a high-frequency word was parafoveally available $[t(23)=6.83$, and $t(23)=6.28$ for the two-wordwindow and full-line conditions, respectively, both $p s<.001$ ] or a low-frequency word was parafoveally available $[t(23)=1.74, p<.09$, and $t(23)=2.93$, $p<.01$, for the two-word-window and full-line conditions, respectively].

To further clarify the critical interaction of word frequency and window size, which showed larger parafoveal preview benefits for high-frequency than for lowfrequency words, an additional analysis was carried out. In some instances, the eyes may have been centered near the end of the word immediately preceding the target, so that word initial letters of the target were projected onto the fovea and the immediately adjoining parafovea prior to fixation of the target; in other instances, the eyes may have been relatively far from the beginning of the target word prior to the fixation of the target so that the word initial letters of the target were projected farther onto a parafoveal region that has a somewhat lower degree of visual acuity. Larger parafoveal preview benefits for high- frequency words than for low-frequency words may have been gained only when the parafoveal target was projected onto the fovea or the immediately adjoining parafovea.

In the additional analysis, first fixations on high- and low-frequency words were divided into two categories: first fixations following saccades that were less than 6.8 character spaces long (the mean saccade length to the target), and first fixations on the target following saccades that were more than 6.8 character spaces long. The results of this analysis are given in Table 2 . As the table indicates, saccade length to the target affected the duration of the first fixation on the target $[F(1,22)=13.15$, $p<.001$ ], so that first fixations were longer after long saccades (longer than 6.8 character spaces) to the target word than after short saccades (shorter than 6.8 character spaces). Saccade length did not, however, qualify the interaction of word frequency and window size. The threeway interaction of these factors did not approach significance $(F<1)$.

The shape of the parafoveal word had no effect on first fixations in either of the two analyses of first fixations. First fixations were $253 \mathrm{msec}$ when letters outside the window were replaced by visually confusable letters and $250 \mathrm{msec}$ when letters outside the window were replaced by $\mathrm{Xs}(F<1)$. None of the interactions with the factor parafoveal mutilation of text outside the window were significant. This corroborates prior research, which shows that word shape is not an effective parafoveal cue (McConkie \& Zola, 1979; Rayner et al., 1980).

We also examined the frequency distributions of first fixations on high- and low-frequency words when parafoveal previews had been available and when parafoveal previews had been denied. These data are shown in Figure 2. The figure shows that when parafoveal previews were available, the distribution for high-frequency words shifted to the left relative to the distribution for lowfrequency words. The distributions in the one-wordwindow conditions revealed that the lack of a parafoveal preview increased the mean fixation duration by shifting the entire distribution to the right (relative to the preview conditions). In essence, the one-word-window condition decreased the probability with which fixations under $250 \mathrm{msec}$ occurred, regardless of the frequency of the fixated word.

Gaze durations. Overall, $10.7 \%$ of all fixations on the target were refixations $(6.8 \%$ on low-frequency words and $3.9 \%$ on high-frequency words) and were included in the computation of gaze durations. In the one-word-window condition, $33 \%$ of all refixations were placed on highfrequency words and $67 \%$ were placed on low-frequency

Table 2

Average First Fixations (in Milliseconds) on Target Words as a Function of Word Frequency, Window Size, and the Size of the Saccade that Preceded the First Fixation

\begin{tabular}{lccccccccc} 
& \multicolumn{3}{c}{ High-Frequency Target } & & \multicolumn{3}{c}{ Low-Frequency Target } & \\
\cline { 2 - 3 } Saccade Size & 1 Word & 2 Words & Full Line & & 1 Word & 2 Words & Full Line & Mean \\
\hline <6.8 Characters & 266 & 226 & 223 & & 263 & 255 & 257 & 248 \\
>6.8 Characters & 294 & 237 & 249 & & 282 & 265 & 253 & 263 \\
\hline
\end{tabular}



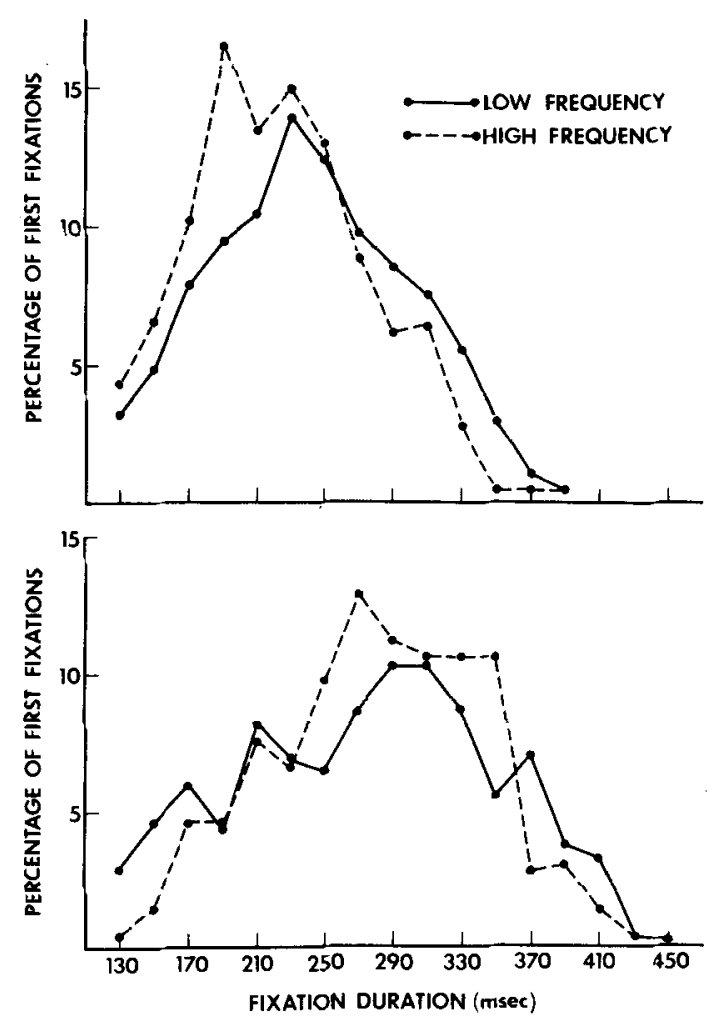

Figure 2. Frequency distribution of first-fixstion durations on highand low-frequency target words, when parafoveal previews were available, as it occurred in the two-word-window and full-line conditions (upper panel) and when parafoveal previews were denied, as it occurred in the one-word-window condition (lower panel). Means of intervals of $\mathbf{2 0}$ msec are displayed.

words; in the parafoveal preview condition, $38 \%$ of all refixations were on high-frequency targets and $62 \%$ were on low-frequency targets. The refixations on highfrequency words in this condition were somewhat shorter $(221 \mathrm{msec})$ than those on low-frequency words $(242 \mathrm{msec})$. When parafoveal previews were provided, the difference in the durations of refixations between highand low-frequency words was marginal (224 and 216 msec, respectively).

This pattern of refixations resulted in a powerful effect of word frequency in the gaze durations, with significantly longer gazes on low-frequency words than on highfrequency words $(272$ and $303 \mathrm{msec})[F(1,20)=63.01$, $p<.001]$. Gaze durations were also affected by window size: $318 \mathrm{msec}$ in the one-word-window condition, $275 \mathrm{msec}$ in the two-word-window condition, and
$273 \mathrm{msec}$ in the full-line condition $[F(2,40)=14.89$, $p<.001]$. The interaction of parafoveal preview and word frequency was not significant $(F<1)$ (see Figure 1).

Comparison of first fixations and gaze. First fixations in the one-word-window condition showed a striking result: when parafoveal previews were denied, first fixations on low-frequency words differed from first fixations on high-frequency words by only $1 \mathrm{msec}$. Refixations in the one-word-window condition were, however, more likely to occur when a low-frequency word was fixated than when a high-frequency word was fixated, which led to longer processing times of a low-frequency word when gazes were computed. The implications of this result are that effects of word frequency on the processing of a word occur relatively late in the fixation time on a word. When no parafoveal preview was provided differences were apparent only when refixations of the word were taken into account. In contrast, refixations on a word were affected by word frequency. The duration of a fixation in reading and the ensuing saccade length thus must have been controlled by different sources of information.

First fixations and gaze durations show similar wordfrequency effects in the parafoveal preview conditions (two-word-window and full-line conditions). This suggests that some processes that were performed during the first fixation of the target when parafoveal previews were denied were performed prior to fixation of the target when parafoveal previews were available.

Saccade length. If readers obtain more effective parafoveal information from a high-frequency parafoveal word than from a low-frequency parafoveal word, they may use this preview information to execute longer saccades to a high-frequency target. This possibility was tested in an additional analysis of saccade length to the high- and low-frequency targets. The effects are shown in Table 3. Only the main effect of window size was significant $[F(2,44)=4.97, p<.01]$. Saccades were shorter when parafoveal previews were denied than when previews were available. Word frequency, in contrast, had no effect on saccade size. Saccade size to high- and low-frequency words was 6.8 character spaces.

\section{Effects of Foveal Word Frequency on Parafoveal Processing}

The previous analyses examined the effects of parafoveal word frequency on parafoveal word processing when the frequency of the foveal word was held constant. Additional analyses of first fixations and gaze on the word following the target (the posttarget word) examined

Table 3

Average Saccade Size (in Character Spaces) as a Function of Parafoveal Mutilation, Word Frequency, and Window Size

\begin{tabular}{|c|c|c|c|c|c|c|c|}
\hline \multirow{2}{*}{$\begin{array}{c}\text { Word } \\
\text { Frequency }\end{array}$} & \multicolumn{3}{|c|}{ Parafoveal Strings of Xs } & \multicolumn{3}{|c|}{ Parafoveally Confusable Letters } & \multirow[b]{2}{*}{ Mean } \\
\hline & 1 Word & 2 Words & Full Line & 1 Word & 2 Words & Full Line & \\
\hline High & 6.4 & 7.2 & 7.2 & 6.8 & 6.5 & 6.7 & 6.8 \\
\hline Low & 6.7 & 7.0 & 7.0 & 6.3 & 6.9 & 7.0 & 6.8 \\
\hline
\end{tabular}


Table 4

First Fixation Durations and Gaze Durations on the Posttarget Word as a Function of the Frequency of the Target Word and Window Size

\begin{tabular}{lcccccccc} 
& \multicolumn{3}{c}{ High Frequency } & & \multicolumn{3}{c}{ Low Frequency } \\
\cline { 2 - 3 } \cline { 7 - 9 } & 1 Word & 2 Words & Full Line & & Word & 2 Words & Full Line \\
\hline First Fixation Duration & 283 & 249 & 245 & & 280 & 257 & 258 \\
Gaze Duration & 331 & 281 & 285 & & 333 & 298 & 299 \\
\hline
\end{tabular}

whether effects of the frequency of the fixated word affected the processing of the parafoveal word, which was held constant.

First fixations. First fixations on posttarget words are shown in Table 4. Only the main effect of window size was significant $[F(2,40)=13.37, p<.001]$. There was also a slight, but not significant, tendency toward shorter posttarget fixations when a posttarget word followed a high-frequency word $[F(1,20)=1.67, p<.2]$. None of the remaining effects were significant.

Gaze durations. Gaze durations are also shown in Table 4. Gaze durations showed, again, prominent window effects $[F(2,40)=11.01, p<.001]$ and a tendency toward shorter gazes on words following a high-frequency word $[F(1,20)=2.72, p<.10]$. Gaze durations also showed a significant interaction of type of parafoveal mutilation outside the window and window size $[F(2,40)=$ $3.62, p<.05]$. A simple comparison of the critical differences in the one-word-window condition $(15 \mathrm{msec})$ was, however, not significant $(t<1)$; that is, the preview of visually confusable letters and of a string of parafoveal Xs did not significantly differ in the gaze durations.

\section{GENERAL DISCUSSION}

There were four major results from the present experiment. First, parafoveal preview and word frequency interacted when first fixations were used as the dependent variable. This interaction ceased to exist when gaze durations were computed. Second, when parafoveal previews were not available, first fixations on a low-frequency target did not differ from first fixations on a high-frequency target. Third, although first fixations were not affected by word frequency when no parafoveal preview was available, lowfrequency words were more likely to be refixated than high-frequency words. Finally, the experiment demonstrated that word frequency affects fixation times on a word when the variability induced by word length is eliminated.

Our first finding supports the position that parafoveal word processing is sensitive to the lexical characteristics of the parafoveal word and that high-frequency parafoveal words are processed more effectively than low-frequency parafoveal words. McClelland and O'Regan (1981) and Balota et al. (1985) found interactive effects of parafoveal preview and predictability of the parafoveal word, and suggested an interactive logogen model of parafoveal word processing to account for their data. According to the model, parafoveally obtained visual information increases the level of activation in all those logogens that are visually similar to the parafoveally obtained preview and decreases the level of activation in logogens that are visually dissimilar; in addition, context is used to activate words that are related to prior context and to inhibit words that are unrelated to prior context. Both sources of activation interact to produce larger parafoveal preview benefits when logogens that are activated by the parafoveal preview are also activated by contextual constraints. A similar interactive mechanism may have produced the interaction of parafoveal preview and word frequency in the present study: parafoveally obtained visual word information might have increased the level of activation in all those logogens that were visually similar to the parafoveally obtained information, and this activation could have interacted with the baseline level of activation, which is assumed to be higher for high-frequency words than for low-frequency words (e.g., Morton, 1969). This interpretation of the results suggests that word frequency and predictability of a parafoveal word exert similar influences on processing, even though effects of prior context are extrinsic to the parafoveal word and effects of parafoveal word frequency are intrinsic to the parafoveal word.

Closer inspection of the data, however, argues against this possibility. The present results show that parafoveal preview and word frequency interacted in first-fixation durations and combined additively when gaze durations were computed. Word frequency thus interacted with those sources of parafoveal information that were available during the first fixation on the target. Balota et al.'s (1985) data showed a different pattern. Parafoveal preview and context interacted in gaze durations, and no significant effects of context emerged when first-fixation durations were computed. Consequently, prior context did not interact with processes that were executed after the target word was initially fixated. Rather, it primarily affected refixations of the target. Assuming that refixations of a word are likely to reflect operations that occur relatively late in the processing of a word, the present results suggest a time-locked sequence of computational events on the parafoveal word. Specifically, effects of parafoveal word frequency precede effects of prior context. Other studies (Balota \& Rayner, 1983; McClelland \& O'Regan, 1981) have shown that context effects accrue over time. For example, Balota and Rayner showed that context and parafoveal preview interacted when the interval between the parafoveal preview and the display of the foveal target was relatively long $(2,000 \mathrm{msec})$. Additive effects of 
context and parafoveal preview were present when a short interval between a parafoveal preview and the following foveal target was used $(250 \mathrm{msec})$. This interpretation of the effects of word frequency and of predictability on parafoveal word processing also agrees with earlier findings that showed, first, that word frequency and predictability tap different stages of processing during the fixation of a word and, furthermore, that these stages are time locked so that effects of word frequency precede effects of predictability (Inhoff, 1984).

The second major finding revealed that when parafoveal previews are withheld, as in the one-word-window condition, the differences in first fixations on high- and lowfrequency words became negligible. This finding contrasts with much recent experimentation that has shown that fixation times (average fixation durations, first fixations) are sensitive to linguistic factors (for a review, see Rayner, 1978). If fixation durations reflect linguistic processing associated with the identification of a word, first fixations on high-frequency words should have been shorter than first fixations on low-frequency words, regardless of whether or not a parafoveal preview was available. The fact that word-frequency effects in the one-word-window condition appeared only when refixations were taken into account could be taken to imply that some fixations in reading are not determined by the linguistic status of the fixated word; rather, on some occasions, the eyes may move to a new location before the meaning of the fixated word is accessed. However, the gaze-duration data suggest that the eyes may not move out of the word in such instances.

Even though first fixations on the target were not affected by word frequency in the one-word-window condition, our third major finding revealed that refixations were more likely to occur when a low-frequency word was fixated than when a high-frequency word was fixated. Thus, even when parafoveal previews were denied, gaze durations were sensitive to linguistic factors. A similar pattern of results appears in the Balota et al. (1985) experiment. In their experiment, first fixations were not affected by the predictability of the fixated word (227 msec on low-predictability words and $223 \mathrm{msec}$ on high-predictability words). Yet the probability of refixating a word was affected by linguistic text characteristics. Specifically, the probability of refixating a highpredictability word was lower than the probability of refixating a low-predictability word, resulting in gaze durations of $232 \mathrm{msec}$ on high-predictability words and $264 \mathrm{msec}$ on low-predictability words. How can cognitive variables affect saccade size, which determines whether the ensuing fixation is a refixation, but not affect fixation durations? If we assume that the decision of when to move the eyes is made prior to the final decision of where to move the eyes ${ }^{1}$ (Becker \& Jurgens, 1979; Morrison, 1984), which may be likely when no effective parafoveal information is obtained, then codes that fail to affect a fixation duration can still affect the length of the following saccade, provided effects of word frequency and predictability become available in the interval that elapses between the decision about when to move and the decision about where to move the eyes.

Decisions about when and where to move the eyes may also account for the differential effects of word frequency on saccades within and across words. Saccades within target words, leading to refixations, were affected by the frequency of the fixated word, so that low-frequency targets were more likely to be refixated than high-frequency targets. Saccade size across words was, in contrast, not affected by the word frequency of the parafoveally available word. Saccades to high- and low-frequency words averaged 6.8 letter spaces each. One way to account for this is to assume that effects of word frequency were present before saccade size was determined only when the target was being fixated; when the target was parafoveally available, effects of word frequency may have followed the final decision of where to move the eyes.

Our fourth major finding demonstrated that highfrequency words receive shorter first fixations than lowfrequency words when parafoveal previews are available. Thus, when parafoveal preview benefits have been obtained, first fixations on a word apparently reflect lexical processing of that word. The difference between high- and low-frequency words in the first-fixation durations occurred even though both word classes were matched for word length (see also Rayner \& Duffy, 1986). Parafoveal information apparently provided sufficient information for fixations to be shorter on high-frequency words than on low-frequency words. Since a parafoveal preview did not affect the size of the saccade to the parafoveal target, parafoveally obtained lexical information appears to be available only after the decisions of when to move and where to move the eyes have been made (see also Pollatsek, Rayner, \& Balota, 1986). Parafoveal information appears to be immediately available at the beginning of the following fixation, so that, for example, effects of word frequency emerge in fixation durations when parafoveal previews are available.

How does parafoveally obtained information affect the processing at the beginning of the following fixation? Four possibilities will be considered. One possibility is that parafoveally obtained word information may be used to narrow the set of possible word candidates that can be encountered on the following fixation. For example, sufficient information may be obtained from the word initial letters of the parafoveal word chest so that the potential word candidates can be limited to chest, cheat, and chart. Information obtained during the following fixation could then be used to narrow the alternatives to a single word candidate. This view predicts that the number of potential word candidates generated from the parafoveal preview should determine the efficiency of parafoveally obtained word information. Specifically, parafoveally available word information that greatly limits the set of potential word candidates should be more effective than parafoveally obtained information that only lousely limits the set of potential word candidates. Lima and Inhoff 
(1985) tested this possibility by comparing preview benefits of parafoveally available word initial letter sequences that either strongly or loosely constrained the number of candidate words that could be encountered on the following fixation. Contrary to the predictions of this type of view, Lima and Inhoff found that constraints imposed by the word initial letter sequence did not affect the benefit obtained from a parafoveal preview.

A second possibility is that some parafoveal words are completely identified after the decision of where to look next has been completed. In this case, the first fixation on the target should be of relatively short duration (Morrison, 1984). The magnitude of the parafoveal preview effect would then be determined by the proportion of trials in which the target was parafoveally identified. Since highfrequency words are more likely to be parafoveally identified, the parafoveal preview benefit will be larger for high-frequency than for low-frequency words. The absence of a frequency effect in the one-word-window condition would be explained if word frequency is needed to supplement the input in the parafovea but not in the fovea.

A third possibility is that perceptual and initial lexical processes are executed twice, once when a word is parafoveally available and then again when it is fixated. Even though parafoveal processing operations are duplicated in the fovea, preview benefits from parafoveally obtained word information can accrue because foveal reprocessing may be more effective than foveal processing when no parafoveal preview has been provided. Since parafoveal preprocessing may progress farther when a high-frequency word is parafoveally available, larger reprocessing benefits should accumulate when a highfrequency word is being fixated.

A final possibility is to assume that parafoveal preview benefits accrue because perceptual and lexical analyses that have been executed parafoveally are omitted during the following fixation. Since parafoveal processing of a high-frequency word progresses farther than the processing of a low-frequency word before an eye movement occurs, larger preview benefits should accrue for highfrequency words. An experiment reported by McConkie et al. (1982) appears to disagree with this possibility. In their experiment, as indicated earlier, they changed certain target words from fixation to fixation as subjects read, so that, for example, the word pears, available on one fixation, was replaced by the word beaks in the same location on the following fixation. If parafoveally initiated word processing was completed during the following fixation, conjunction errors of parafoveal and foveal words should have occurred; that is, subjects should, occasionally, have perceived the words peaks or bears. Conjunctions of this type were, however, rarely reported by subjects. However, McConkie et al.'s (1982) study showed only small (10- to $12.5-\mathrm{msec})$ preview effects, which were not statistically significant. Since parafoveal preview effects were small, conjunction errors should be rare or absent. McConkie et al.'s data thus do not rule out the pos- sibility that parafoveally initiated visual and lexical processes are not duplicated during the following fixation. Thus, the idea that foveal processing resumes the processing that was initiated parafoveally is still viable as an explanation of parafoveal preview benefits.

The last three of the four possibilities that we have discussed for how parafoveally obtained information affects processing at the beginning of a fixation seem feasible. It should be noted that these possibilities need not be mutually exclusive. It could be that on some fixations a highfrequency parafoveal word is completely identified after the decision to move the eyes has been made; on other fixations, the reader may obtain, from the parafoveal preview, partial word information that is reprocessed or integrated during the following fixation.

Finally, our finding that high-frequency words received shorter fixations (when previews were available) than lowfrequency words also pertains to an issue raised by Kliegl et al. (1982). Specifically, Kliegl et al. pointed out that earlier studies that had shown effects of word frequency on fixation time confounded word length and word frequency. Kliegl et al. used hierarchical regression analyses to separate effects of word length and word frequency on fixation times during reading. They found that word frequency accounted for only $3 \%$ of the variance when word length was entered before word frequency in the regression analysis. The substantial word-frequency effects in the present study and in the recent study by Rayner and Duffy (1986) suggest that this computation underestimates the effect of word frequency on fixation times when parafoveal previews are available; our results appear to be in closer agreement with Kliegl et al.'s additional finding that word frequency accounted for a more substantial amount of variance when it was entered first into the regression analysis. Additional experimentation that manipulates word frequency and word length indepenently will be needed to delineate the specific contributions of word frequency and word length on fixation times in reading.

\section{REFERENCES}

BalotA, D. A., \& Chumbley, J. I. (1984). Are lexical decisions a good measure of lexical access? The role of word frequency in the neglected decision stage. Joumal of Experimental Psychology: Human Perception \& Performance, 10, 340-357.

Balota, D. A., Pollatsek, A., \& Rayner, K. (1985). The interaction of contextual and parafoveal visual information during eye fixations in reading. Cognitive Psychology, 17, 364-390.

Balota, D. A., \& RA YNeR, K. (1983). Parafoveal visual information and semantic contextual constraints. Joumal of Experimental Psychology: Human Perception \& Performance, 5, 726-738.

BeCKer, W., JuRgens, R. (1979). An analysis of the saccadic system by means of double step stimuli. Vision Research, 19, 967-983.

Ehrlich, S. F., \&AYNER, K. (1981). Context effects on word perception and eye movements in reading. Journal of Verbal Leaming \& Verbal Behavior, 20, 641-655.

Gernsbacher, M. A. (1984). Resolving 20 years of inconsistent interactions between lexical familiarity and orthography, concreteness, and polysemy. Joumal of Experimental Psychology: General, 113, 256-281. 
Hogaboam, T. W. (1983). Reading patterns in eye movement data. In K. Rayner (Ed.), Perceptual and language processes. New York: Academic Press.

HUEY, E. B. (1968). The psychology and pedagogy of reading. Boston: MIT Press. (Original work published 1908)

INHOFF, A. W. (1984). Two stages of word processing during eye fixations in the reading of prose. Journal of Verbal Learning \& Verbal Behavior, 23, 612-624.

INHOFF, A. W. (1985). The effect of factivity on lexical retrieval and postlexical processing during eye fixations in reading. Journal of Psycholinguistic Research, 14, 45-56.

Just, M. A., \& Carpenter, P. A. (1980). A theory of reading: From eye fixations to comprehension. Psychological Review, 87, 329-354.

KLIEGL, R., OLSON, R. K., \& DAVIDSON, B. J. (1982). Regression analyses as a tool for studying reading processes: Comments on Just and Carpenter's eye fixation theory. Memory \& Cognition, 10, 287-296.

KuČERA, H., FRANCIS, W. N. (1967). Computational analysis of present day American English. Providence, RI: Brown University Press.

LimA, S. D., \& INHOFF, A. W. (1985). Lexical access during eye fixations in reading: Effects of word initial letter sequence. Journal of Experimental Psychology: Human Perception \& Performance, 11, 272-283.

McClelland, J. L., \& O'Regan, K. (1981). Expectations increase benefits derived from parafoveal visual information in reading words aloud. Joumal of Experimental Psychology: Human Perception \& Performance, 7, 634-644.

MCCONKIE, G. W., RAYNER, K. (1975). The span of the effective stimulus during a fixation in reading. Perception \& Psychophysics, 17, 578-587.

MCConkIE, G. W., \& ZoLA, D. (1979). Is visual information integrated across successive fixations in reading? Perception \& Psychophysics, 25, 221-224.

McConkie, G. W., Zola, D., Blanchard, D. E., * Wolverton, G. S. (1982). Perceiving words during reading: Lack of facilitation from prior peripheral exposure. Perception \& Psychophysics, 32, 272-281.

Morrison, R. E. (1984). Manipulation of stimulus onset delay in reading: Evidence for parallel programming of saccades. Journal of Experimental Psychology: Human Perception \& Performance, 10, $667-682$.

Morton, J. (1969). Interaction of information in word recognition. Psy chological Review, 76, 165-178.
Pollatsek, A., \& RaYNer, K. (1982). Eye movement control in reading: The role of word boundary. Journal of Experimental Psychology: Human Perception \& Performance, 8, 817-833.

Pollatsek, A., Rayner, K., \& Balota, D. A. (1986). Inferences about eye movement control from the perceptual span in reading. Perception \& Psychophysics, 40, 123-130.

RAYNER, K. (1977). Visual attention in reading: Ey z movements reflect cognitive processes. Memory \& Cognition, 4, 443-451.

RAYNER, K. (1978). Eye movements in reading and information processing. Psychological Bulletin, 85, 618-660.

RAYNER, K. (1979). Eye guidance in reading. Fixation locations within words. Perception, 8, 21-30.

Rayner, K., Balota, D. A., \& Pollatsek, A. (in press). Against parafoveal semantic preprocessing during eye fixations in reading. Canadian Journal of Psychology.

RAYNer, K., Bertera, J. H. (1979). Reading without a fovea. Science, 206, 468-469.

RAYNER, K., DUFFY, S. A. (1986). Lexical complexity and fixation times in reading: Effects of word frequency, verb complexity, and lexical ambiguity. Memory \& Cognition, 14, 191-201.

RaYNer, K., INhoff, A. W., Morrison, R. E., SlowiaczeK, M. L., \& BERTERA, J. H. (1981). Masking of foveal and parafoveal vision during eye fixations in reading. Journal of Experimental Psychology: Human Perception \& Performance, 7, 167-181.

RAYNer, K., MCCONKIE, G. W., \& Zola, D. (1980). Integrating information across eye movements. Cognitive Psychology, 12, 206-226.

Rayner, K., Well, A. D., Pollatsek, A., Bertera, J. H. (1982). The availability of useful information to the right of fixation in reading. Perception \& Psychophysics, 31, 537-550.

WhALEY, C. P. (1978). Word-nonword classification time. Journal of Verbal Leaming \& Verbal Behavior, 17, 143-154.

\section{NOTE}

1. It is possible that a preliminary decision on where to move the eyes occurs before the final decision on where to move the eyes (Pollatsek \& Rayner, 1982).

(Manuscript received April 8, 1986; revision accepted for publication September 19,1986.) 